Revue internationale P.M.E.

Économie et gestion de la petite et moyenne entreprise

\title{
La planification du processus de transmission dans les PME québécoises
}

\section{Louise St-Cyr et Francine Richer}

Volume 18, numéro 3-4, 2005

URI : https://id.erudit.org/iderudit/1008482ar

DOI : https://doi.org/10.7202/1008482ar

Aller au sommaire du numéro

\section{Éditeur(s)}

Presses de l'Université du Québec

ISSN

0776-5436 (imprimé)

1918-9699 (numérique)

Découvrir la revue

\section{Citer cet article}

St-Cyr, L. \& Richer, F. (2005). La planification du processus de transmission dans les PME québécoises. Revue internationale P.M.E., 18(3-4), 51-71. https://doi.org/10.7202/1008482ar

\section{Résumé de l'article}

À partir d'une enquête menée auprès de 115 dirigeants d'entreprises québécoises ayant complété avec succès une transmission d'entreprise, cette recherche tente de cerner l'étendue de la planification du processus de transmission de la direction et d'évaluer la conformité du comportement des répondants aux « meilleures pratiques » en ce domaine. Les activités de planification examinées se rapportent principalement aux critères de choix du successeur, à sa préparation ainsi qu'au désengagement du prédécesseur. Des éléments contextuels, comme la mise en place d'un conseil d'administration et d'un conseil de famille, sont également étudiés.

Les résultats révèlent que les activités relatives à l'établissement de critères de choix du successeur et à leur communication et celles se rapportant à la préparation du successeur sont nombreuses et fidèles aux « meilleures pratiques ". Tel n'est pas le cas des activités reliées au désengagement du prédécesseur, à la présence de structures comme le conseil d'administration et le conseil de famille et à l'existence de documents écrits sur la vision d'avenir de l'entreprise. Deux éléments peuvent expliquer cette non-conformité aux " meilleures pratiques » chez des entreprises ayant réussi. Dans certains cas, des activités de compensation semblent avoir pris place et, dans d'autres, un effet de taille se manifeste, les petites entreprises étant celles qui s'y conforment le moins.

Cette étude, la première réalisée sur un si grand nombre de sujets québécois, fait ressortir la souplesse dont peuvent faire preuve les entreprises dans la gestion de la relève à la haute direction. Elle souligne aussi la nécessité de tenir compte du contexte de l'entreprise, notamment de sa taille, de façon à relativiser la liste des " meilleures pratiques ». L’article conclut sur la nécessité de parfaire un modèle intégrateur des activités de planification susceptible d'être testé en contexte de PME de façon à distinguer les activités qui sont essentielles de celles qui peuvent faire l'objet d'une certaine flexibilité.
Ce document est protégé par la loi sur le droit d'auteur. L'utilisation des services d’Érudit (y compris la reproduction) est assujettie à sa politique d'utilisation que vous pouvez consulter en ligne.

https://apropos.erudit.org/fr/usagers/politique-dutilisation/ 


\title{
La planification du processus de transmission dans les PME québécoises
}

\author{
Louise ST-CYR \\ Francine RICHER \\ Chaire de développement et de relève de la PME \\ École des Hautes Études commerciales de Montréal
}

\section{MOTS CLÉS}

Relève - Transmission - Planification - Successeur
Prédécesseur - Facteur de succès - PME - Formation - Québec

\section{RÉSUMÉ}

À partir d'une enquête menée auprès de 115 dirigeants d'entreprises québécoises ayant complété avec succès une transmission d'entreprise, cette recherche tente de cerner l'étendue de la planification du processus de transmission de la direction et d'évaluer la conformité du comportement des répondants aux «meilleures pratiques" en ce domaine. Les activités de planification examinées se rapportent principalement aux critères de choix du successeur, à sa préparation ainsi qu'au

\section{LES AUTEURES}

LOUISE ST-CYR, FCA, est titulaire de la Chaire de développement et de relève de la PME, professeure titulaire de finance et directrice du Groupe Femmes, gestion et entreprises à l'Ecole des Hautes Études commerciales de Montréal. Ses recherches sont axées sur le phénomène de la transmission des PME, l'évaluation des entreprises à fort potentiel de croissance et l'entrepreneuriat féminin. Parmi ses récentes publications, mentionnons Préparer la relève neuf études de cas sur l'entreprise au Québec, 2003 et PME: conseils et solutions, 2005. Adresse: Chaire de développement et de relève de la PME, HEC Montréal, 3000, chemin de la Côte-Sainte-Catherine, Montréal, Québec, H3T 2A7. Téléphone: (514) 340-6015, télécopieur: (514) 340-3825. Courriel: <louise.st-cyr@hec.ca>

FRANCINE RICHER, M.A. (en éducation), est membre associée de la Chaire de développement et de relève de la PME. Elle s'intéresse depuis plus de quinze ans à la dynamique des carrières, particulièrement aux cheminements diversifiés des successeurs, filles et fils, ainsi qu'au processus de transmission des entreprises. Analyste et rédactrice de cas, elle est coauteure avec Louise St-Cyr de Préparer la relève - neuf études de cas sur l'entreprise au Québec, 2003, et elle a collaboré à l'édition de PME: conseils et solutions, 2005. Adresse: Chaire de développement et de relève de la PME, HEC Montréal, 3000, chemin de la CôteSainte-Catherine, Montréal, Québec, H3T 2A7. Téléphone: (450) 663-7373, télécopieur: (514) 340-3825. Courriel: <francine.richer@hec.ca>.

(C) 2005 - Presses de l'Université du Québec

Édifice Le Delta I, 2875, boul. Laurier, bureau 450, Sainte-Foy, Québec G1V 2M2 • Tél.: (418) 657-4399 - www.puq.ca

Tiré de: Revue internationale P.M.E., vol. 18, nos $3-4$, sous la direction de Louis Raymond - PME1803N

Tous droits de reproduction, de traduction et d'adaptation réservés 
désengagement du prédécesseur. Des éléments contextuels, comme la mise en place d'un conseil d'administration et d'un conseil de famille, sont également étudiés.

Les résultats révèlent que les activités relatives à l'établissement de critères de choix du successeur et à leur communication et celles se rapportant à la préparation du successeur sont nombreuses et fidèles aux «meilleures pratiques». Tel n'est pas le cas des activités reliées au désengagement du prédécesseur, à la présence de structures comme le conseil d'administration et le conseil de famille et à l'existence de documents écrits sur la vision d'avenir de l'entreprise. Deux éléments peuvent expliquer cette non-conformité aux «meilleures pratiques» chez des entreprises ayant réussi. Dans certains cas, des activités de compensation semblent avoir pris place et, dans d'autres, un effet de taille se manifeste, les petites entreprises étant celles qui s'y conforment le moins.

Cette étude, la première réalisée sur un si grand nombre de sujets québécois, fait ressortir la souplesse dont peuvent faire preuve les entreprises dans la gestion de la relève à la haute direction. Elle souligne aussi la nécessité de tenir compte du contexte de l'entreprise, notamment de sa taille, de façon à relativiser la liste des «meilleures pratiques». L'article conclut sur la nécessité de parfaire un modèle intégrateur des activités de planification susceptible d'être testé en contexte de PME de façon à distinguer les activités qui sont essentielles de celles qui peuvent faire l'objet d'une certaine flexibilité.

\begin{abstract}
Based on a survey of 115 directors of Quebec companies that have been successfully transferred at least once, this research attempts to explore the extent to which the transfer of management process was planned and to evaluate the conformity of the respondents'behaviour to the "best practices". The aspects of planning that were examined are the set up of criteria for the successor selection, the development of successor and the incumbent phase out and new role. Other contextual elements that were considered are the presence of a board of directors and a family council.

The findings reveal that planning activities related to the set up of criteria for successor selection and the development of successor are numerous and conform to "best practices". This is not the case for the incumbent phase out and new role, the presence of a board of directors, a family council and a written strategic plan. Two reasons might explain how come some enterprises could succeed in transferring their company even though they have not conformed to "best practices". First, in some cases, they might have compensated with other types of activities. Second, the size of the company seems to impact on some of the results, the smaller enterprises being the one that conform the least to some of the "best practices".

This study, the first to be conducted on such a large number of subjects in Quebec, highlights not only the flexibility of business owners when planning the succession process but also the importance of taking the context of the business into consideration when establishing "best practices". The article concludes on the necessity to perfect an integrative model of succession planning that can be tested in an SME context and that can depart the really essential activities from those that are less essential.
\end{abstract}




\section{RESUMEN}

En base a una encuesta en la cual participaron 115 directivos empresariales de Quebec que llevaron exitosamente a cabo la transmisión de su negocio, la presente investigación trata de analizar en qué medida fue planificado dicho proceso de transmisión de la dirección y evaluar hasta qué punto los encuestados actuaron conforme a las «mejores prácticas» recomendadas en estos casos. Se examinan particularmente los aspectos de la planificación relacionados con los criterios establecidos para la elección del sucesor, la preparación de éste para las nuevas tareas y la separación gradual del titular, así como elementos contextuales, tales la creación de un consejo de administración o de un consejo familiar.

Los resultados demuestran que las actividades relacionadas con el establecimiento de criterios para la elección del sucesor y su divulgación, así como las vinculadas a la preparación del sucesor son numerosas y fieles a las «mejores prácticas", lo que no se puede decir de las realizadas en torno de la separación progresiva del titular predecesor, la presencia de estructuras como un consejo de administración o un consejo de familia o la existencia de escritos sobre la visión para el futuro de la empresa. Dos elementos pueden explicar esta falta de conformidad con las «mejores prácticas» por parte de las empresas exitosas. En ciertos casos, actividades de compensación parecen haberse puesto en pie $y$, en otros casos, el tamaño de la empresa parece desempeñar un papel evidente, siendo las más pequeñas las que menos se conforman con las «mejores prácticas».

Este estudio, el primero en ser realizado con un número tan grande de sujetos en Quebec, destaca no solo la flexibilidad que poseen los propietarios de empresa al planificar el proceso de sucesión, sino también la importancia de tomar en cuenta el contexto de la empresa al establecer las «mejores prácticas». En la conclusión se plantea la necesidad de perfeccionar un modelo integrado para la planificación de la sucesión que pueda ser ensayado en el ámbito de las PyMEs y que pueda distinguir las actividades verdaderamente esenciales $y$ las que se prestan a mayor flexibilidad.

\section{ZUSAMMENFASSUNG}

Anhand einer Untersuchung bei 115 Unternehmern aus Québec, die mit Erfolg die Nachfolge im Unternehmen vollzogen hatten, versucht die vorliegende Forschungsarbeit den Umfang der Nachfolgeplanung zu bestimmen und zusätzlich das Vorgehen der Befragten mit den "best practices" aus diesem Bereich zu vergleichen. Die untersuchten Vorgehensweisen der Nachfolgeplanung beziehen sich hauptsächlich auf die Kriterien zur Auswahl des Nachfolgers, seiner Vorbereitung sowie auf den Rückzug des Vorgängers. Ebenfalls untersucht wurden die notwendigen kontextuellen Elemente, wie beispielsweise die Wahl eines Verwaltungs- oder Familienrates.

Die Ergebnisse der Untersuchung zeigen auf, dass die Vorgehensweisen zur Ausarbeitung und Kommunikation der Auswahlkriterien sowie zur Vorbereitung der Nachfolger stark den "best practices" entsprechen. Diese grosse Übereinstimmung mit den "best practices" ist andernorts nicht gegeben. Besonders bei Aktivitäten in Zusammenhang mit dem Rückzug des Unternehmers, dem Umgang mit Strukturen wie Verwaltungs- oder Familienrat und mit der schriftlichen Kommunikation

Revue internationale P.M.E., vol. 18, ${ }^{\text {os }} 3-4,2005$

(C) 2005 - Presses de l'Université du Québec

Édifice Le Delta I, 2875, boul. Laurier, bureau 450, Sainte-Foy, Québec G1V 2M2 • Tél.: (418) 657-4399 - www.puq.ca

Tiré de: Revue internationale P.M.E., vol. 18, nos $3-4$, sous la direction de Louis Raymond - PME1803N

Tous droits de reproduction, de traduction et d'adaptation réservés 
der Unternehmensvision besteht grosses Potential. Zwei Elemente können diese bestehende Diskrepanz zu den "best practices" erklären. In einigen Fällen haben Kompensationsaktivitäten stattgefunden, in anderen Fällen hatten Grösseneffekte ihren Einfluss, so entsprechen kleine Unternehmen den "best practices" tendenziell weniger.

Unsere Studie, die erste in diesem Umfang im Raum Québec, zeigt auf, mit welcher Flexibilität die Ablösung von Führungskräften in Unternehmen vollzogen wird. Darüber hinaus wird die Wichtigkeit hervorgehoben, die Rahmenbedingungen und Situation der Unternehmung (z.B. die Grösse) zu berücksichtigen, um die Vorgaben der "best practices" relativieren zu können. Der Artikel schliesst mit dem Hinweis auf die Notwendigkeit der Erstellung eines integrativen Planungsmodells für die Nachfolgeregelungen, das im KMU-Umfeld getestet werden kann und die Unterscheidung von zwingenden und Aktivitäten, die mit einer gewissen Flexibilität gehandhabt werden können, erlaubt.

\section{Introduction}

La difficulté, voire l'incapacité des entreprises à réaliser avec succès le transfert intergénérationnel est une situation préoccupante si l'on considère l'incidence que la disparition d'une entreprise a sur l'économie. Dans une étude encore largement citée aujourd'hui, Ward (1987) a démontré que près de $70 \%$ des entreprises familiales ne survivent pas plus de deux générations et, à peine $10 \%$ à $15 \%$, plus de trois générations. Les causes expliquant un tel phénomène sont nombreuses et certaines résident vraisemblablement dans le taux normal d'attrition des entreprises à travers le temps ${ }^{1}$. Cependant, même si le côté alarmant de ces statistiques peut être mis en doute (Aronoff, 2001), il semble tout de même que certaines disparitions pourraient être évitées, et ce, grâce à une meilleure planification du processus de transmission (Sharma et al., 2001; Davis et Harveston, 1988; Ward, 1987).

Le processus de transmission et sa planification ont retenu l'attention de nombreux chercheurs depuis les 20 dernières années. Plusieurs d'entre eux ont travaillé à documenter ce processus, notamment à en établir les principales composantes et les éléments pouvant faire l'objet d'une planification. Partant de l'hypothèse que la planification du processus est souhaitable puisqu'elle en augmente les chances de succès, d'autres chercheurs ont tenté de déterminer les facteurs qui incitent les dirigeants à entreprendre des activités de planification. Enfin, certains ont dégagé, à partir de l'étude de cas de transmissions réussies, les bonnes pratiques à recommander en cette matière.

1. Ces disparitions peuvent s'expliquer par des fusions et acquisitions d'entreprises ou résulter de fermetures volontaires. 
La recherche présentée dans cet article se situe dans la continuité de ces dernières études. À partir d'un échantillon d'entreprises québécoises ayant complété avec succès un transfert intergénérationnel, l'étude procède à l'identification et à l'analyse des activités réalisées par ces entreprises dans le cadre de la planification du processus de transmission de la direction. L'article se divise en quatre parties: la première section présente le cadre conceptuel; la deuxième, le cadre opératoire; la troisième, les résultats; la dernière section ouvre sur les avenues futures de recherche.

\section{Cadre conceptuel}

\subsection{Un processus}

La majorité des auteurs ont présenté la transmission d'une entreprise, non pas comme un événement unique se produisant à un moment précis, mais plutôt comme un long processus divisé en un certain nombre d'étapes durant lesquelles le successeur est amené à accroître son rôle dans l'entreprise tandis que le prédécesseur s'en désengage progressivement (Longenecker et Schoen, 1978; McGivern, 1978; Churchill et Hatten, 1987; Handler, 1990; Hugron, 1991; Murray, 2003). Hugron (1991) fut le premier à reconnaître explicitement deux dimensions à ce processus, soit le transfert de la direction et celui de la propriété. Chacune de ces dimensions est elle-même divisée en quatre étapes. L'étape de l'incubation, celle du choix du ou des successeurs, le règne conjoint et l'étape du désengagement du prédécesseur subdivisent la dimension du transfert de la direction. La fixation d'un modèle de dévolution de la propriété, la consultation d'experts, le choix des solutions disponibles et, finalement, la sanction des ententes et leur mise en œuvre constituent les étapes du transfert de propriété. Handler (1990) propose, pour sa part, de concevoir la transmission d'une entreprise comme un processus d'ajustement mutuel de rôles entre le prédécesseur et le successeur dans lequel, contrairement aux auteurs cités précédemment, ce ne sont pas les étapes du processus qui influencent les rôles et les interactions entre les acteurs, mais plutôt l'évolution de ces rôles et interactions qui en détermine les différentes phases. Le fait que tous s'accordent pour parler d'un processus vient renforcer l'importance de sa planification. La séparation du processus en dimensions et en étapes facilite l'identification des éléments qui s'intègrent dans une démarche planifiée.

\subsection{La planification}

Comme le font remarquer Sharma, Chua et Chrisman (2003), bien que la planification de la transmission de la direction puisse être définie de façon unidimensionnelle, la compréhension du phénomène du transfert intergénérationnel gagne à donner au concept de planification un sens plus large qui englobe l'ensemble

Revue internationale P.M.E., vol. 18, ${ }^{\text {os }} 3-4,2005$

(C) 2005 - Presses de l'Université du Québec

Édifice Le Delta I, 2875, boul. Laurier, bureau 450, Sainte-Foy, Québec G1V 2M2 • Tél.: (418) 657-4399 - www.puq.ca

Tiré de: Revue internationale P.M.E., vol. $18, \mathrm{n}^{\text {os }} 3-4$, sous la direction de Louis Raymond • PME1803N

Tous droits de reproduction, de traduction et d'adaptation réservés 
des activités qui sont entreprises, tout au long du processus, dans le but ultime de réaliser la transmission. Cette conception globale de la planification est relevée aussi par Lansberg (1988) lorsqu'il définit la planification comme étant «[les activités] de préparation nécessaires pour assurer l'harmonie dans la famille et la continuité de l'entreprise à la prochaine génération. Ces préparatifs doivent être pensés en fonction des besoins futurs à la fois de la famille et de l'entreprise ${ }^{2}$ ».

En se basant sur le modèle du processus de Hugron (1991), on peut classer les activités reliées à la planification du transfert de direction en trois catégories, soit celles relatives à la préparation du successeur (phases d'incubation et du règne conjoint), celles qui sont liées au choix du successeur (phase du choix du successeur) et celles qui touchent le rôle du prédécesseur une fois qu'il a pris sa décision et qu'il cède la direction de l'entreprise (phase de désengagement du prédécesseur). Dans leur article étudiant la planification de la succession à la lumière de la théorie du comportement planifié, Sharma, Chua et Chrisman (2003) ajoutent une quatrième catégorie d'activités, soit celles reliées à l'existence d'un plan stratégique ou d'une vision de l'entreprise une fois que le successeur a pris les rênes de l'entreprise. Pour chacune de ces quatre catégories, les recherches antérieures permettent d'identifier, non seulement les activités qui peuvent faire l'objet d'une planification, mais également les pratiques à adopter (les «meilleures pratiques») qui maximisent les chances de succès de la transmission.

Les activités relatives à la préparation et au développement du successeur sont jugées primordiales par plusieurs (Ward, 1987; Cabrera-Suárez, De Saa-Pérez et García-Almeida, 2001). Elles incluent l'atteinte d'un niveau de scolarité suffisant, l'expérience prise à l'extérieur de l'entreprise, l'expérience prise à l'interne (niveau du poste obtenu à l'entrée et durée) et le mentorat ainsi que d'autres formes d'initiation à la fonction de direction, la responsabilité de mandats spéciaux, par exemple. La majorité des auteurs conclut à l'avantage d'avoir fait des études prolongées (Goldberg, 1996; Morris et al., 1997). Le côté positif de l'expérience prise à l'extérieur de l'entreprise est mis en lumière par plusieurs également (Barach et Gantisky, 1995; Barach et al., 1988; Ward, 1987). Il en est de même de l'accession graduelle à la direction (Barach et al., 1988; Morris et al., 1997).

En ce qui a trait au choix du successeur, les activités de planification comprennent notamment l'établissement de critères de choix et leur communication aux principaux intéressés. Il est généralement reconnu que cette communication à l'avance des critères de choix constitue une bonne pratique (Ward, 1987).

Enfin, pour ce qui est du rôle du prédécesseur après la transmission, le fait d'avoir prévu un rôle satisfaisant pour ce dernier et d'avoir fixé une date pour son départ à la retraite, comptent parmi les décisions associées à des activités de plani-

2. Traduction libre.

Revue internationale P.M.E., vol. 18, n ${ }^{\text {os }} 3-4,2005$

(C) 2005 - Presses de l'Université du Québec

Édifice Le Delta I, 2875, boul. Laurier, bureau 450, Sainte-Foy, Québec G1V 2M2 - Tél.: (418) 657-4399 - www.puq.ca

Tiré de: Revue internationale P.M.E., vol. 18, nos $3-4$, sous la direction de Louis Raymond - PME1803N

Tous droits de reproduction, de traduction et d'adaptation réservés 
fication. Parce que ces activités témoignent, jusqu'à un certain point, de l'intention des prédécesseurs de se désengager véritablement et que l'intention de se désengager est une des conditions pour une transmission réussie (Sharma et al., 2001; Cadieux et Lorrain, 2004), elles peuvent être qualifiées de bonnes pratiques.

Quant à l'existence d'une vision de l'entreprise une fois le successeur en place, elle est assurée par l'existence de documents écrits qui en témoignent. La rédaction de ces documents est, sans contredit, une activité de planification reconnue comme un autre facteur de succès de la transmission (Cliffe, 1998).

À l'ensemble de ces activités qui révèlent un souci de planification de la transmission, on peut ajouter les indicateurs suivants: la présence d'un conseil d'administration et d'un conseil de famille. La présence de ces deux structures a été jugée salutaire par plusieurs auteurs (Malone, 1989; Lansberg, 1988) quoique le rôle du conseil de famille dans le cadre particulier de la planification ait été peu abordé (Le Breton-Miller et Miller, 2004).

En regroupant les activités relevées ci-dessous conformément au modèle intégrateur des «meilleures pratiques» en matière de transmission de Le Breton-Miller et Miller (2004), on obtient un schéma des activités de planification du processus de transmission (voir la figure 1). Il se divise en cinq catégories. Trois de ces catégories font référence au processus de succession lui-même. Ce sont, en séquence: 1) l'établissement de règles de base reliées à la planification du processus, 2) la préparation du successeur et 3) le désengagement du prédécesseur. Deux autres catégories renvoient à des éléments contextuels: 4) le contexte de l'entreprise et 5) le contexte familial. Le schéma présenté ici est partiel et ne prétend pas être exhaustif au regard de toutes les activités de planification qui peuvent prendre place avant et pendant le processus de transmission. Il vise celles ayant reçu, jusqu'à présent, le plus d'attention dans la littérature sur les entreprises familiales (Le Breton-Miller et Miller, 2004, p. 312) 3 $^{3}$ Ainsi, les activités de planification du processus de transmission de la propriété, bien qu'importantes (File et Prince, 1996; BDPME, 1998), n'ont pas été incluses dans ce schéma, cet article étant avant tout centré sur la planification du processus de transmission de la direction.

Dans la continuité d'une étude exploratoire menée au Québec sur les stratégies de succès du processus de transmission en contexte de PME (St-Cyr et Richer, 2002) et à la lumière du modèle ci-dessous, l'objectif de cet article est d'examiner l'expérience d'un groupe d'entreprises québécoises ayant réalisé avec succès une transmission intergénérationnelle. Il tente de répondre aux deux questions suivantes.

3. Ces auteurs présentent d'ailleurs, dans ce même article, une version plus complète de ce modèle.

Revue internationale P.M.E., vol. 18, ns 3-4, 2005

(C) 2005 - Presses de l'Université du Québec

Édifice Le Delta I, 2875, boul. Laurier, bureau 450, Sainte-Foy, Québec G1V 2M2 • Tél.: (418) 657-4399 - www.puq.ca

Tiré de: Revue internationale P.M.E., vol. 18, nos $3-4$, sous la direction de Louis Raymond - PME1803N

Tous droits de reproduction, de traduction et d'adaptation réservés 
Figure 1

Activités de planification du processus de la direction

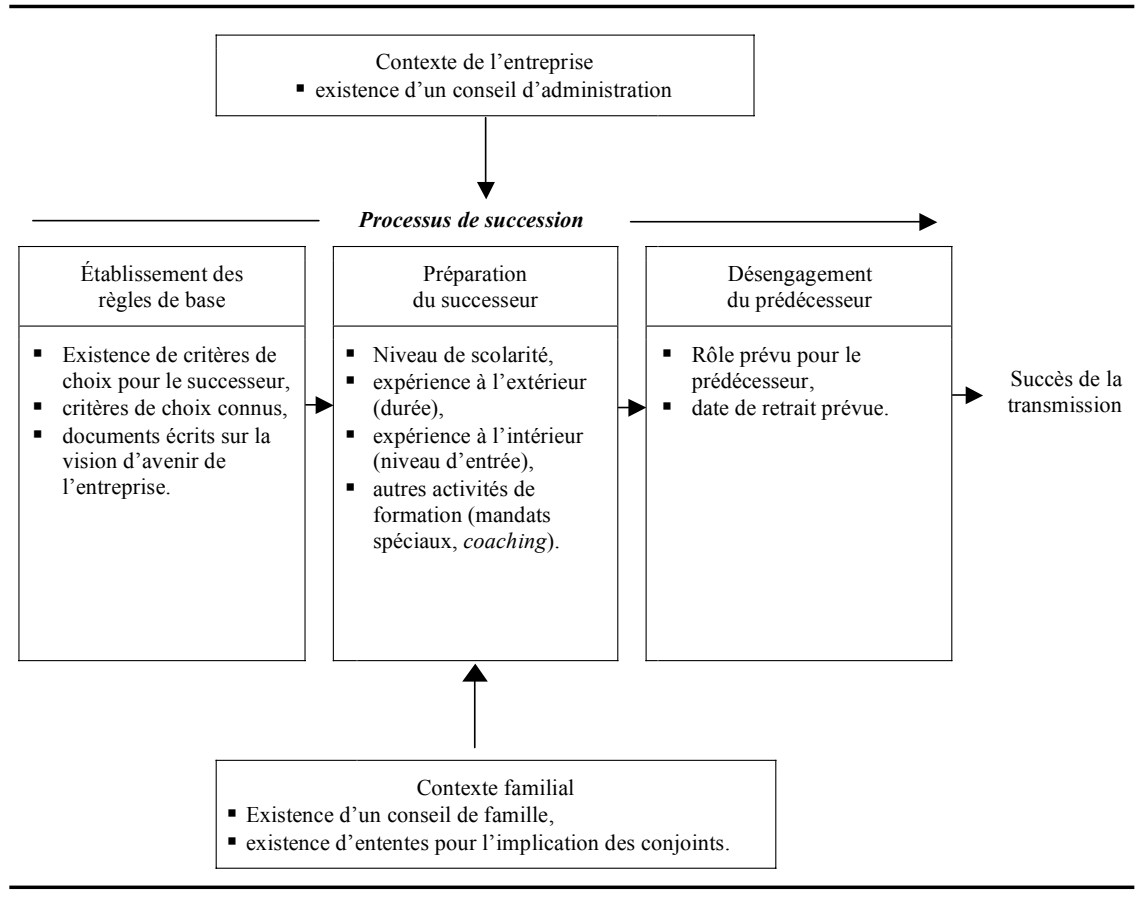

Source: Adaptation de Le Breton-Miller et Miller, 2004 - version simplifiée.

1. Quelles sont les activités de planification mises en œuvre pour transmettre la direction de la PME familiale au Québec?

2. Comment ces activités se comparent-elles aux «meilleures pratiques»?

Jusqu'à présent, peu de recherches ont été menées au Québec sur le phénomène de la transmission d'entreprise auprès d'un échantillon d'assez grande taille en dépit du fait que ce thème est incontestablement d'actualité. Un sondage réalisé en 2000 auprès de propriétaires de PME du Québec révélait que 56\% des dirigeants envisageaient se retirer des affaires d'ici cinq à dix ans et que $66 \%$ d'entre eux souhaitaient que l'entreprise demeure dans la famille (Campbell Expertise Marketing, 2000). De plus, comme le faisaient remarquer Le Breton-Miller et Miller (2004), plusieurs articles publiés sur la transmission d'entreprises et les «meilleures pratiques» sont de type «anecdotique, c'est-à-dire qu'ils reposent principalement sur l'opinion ou l'expérience de l'auteur, habituellement un prati- 
cien ou un consultant et s'appuient sur quelques exemples très riches ${ }^{4} »$ (p. 319). Dans cette perspective, le présent article contribue à la validation empirique de la planification du processus de transmission et de quelques-unes des «meilleures pratiques» en ce domaine.

\section{Cadre opératoire}

\subsection{Constitution de l'échantillon}

L'échantillon a été constitué à partir du répertoire du Centre de recherche industrielle du Québec (CRIQ) ${ }^{5}$. Afin de constituer l'échantillon de départ, les critères de sélection suivants ont été retenus : entreprise embauchant entre 10 et 499 employés, chiffre d'affaires supérieur à un million de dollars et fondation antérieure à 1974. Les deux premiers critères visaient à inclure des PME de taille suffisante pour que le processus de relève présente un certain degré de complexité. Le critère de l'année de fondation a été ajouté afin d'augmenter les chances qu'un premier transfert ait été réalisé. L'échantillon ainsi obtenu comprenait toutes les entrées au fichier du CRIQ qui correspondaient à ces critères et comptait 2003 entreprises: 1243 entreprises manufacturières, 560 grossistes et distributeurs et 200 entreprises de services. Une première série d'appels téléphoniques a permis d'éliminer 933 entreprises (entreprises de première génération, filiales ou divisions d'une grande entreprise, doublons, entreprises disparues, etc.). L'échantillon fut ainsi réduit à 1070 entreprises. Une deuxième série d'appels, dont l'objectif était de solliciter la participation du dirigeant de l'entreprise à l'enquête, a finalement permis de joindre 173 sujets, prédécesseurs et successeurs. L'écart s'explique ainsi: 700 dirigeants n'ont pu être joints personnellement, malgré trois tentatives, et 197 ont refusé de participer.

Les résultats présentés dans cet article se rapportent à l'expérience de 115 de ces 173 sujets, soit uniquement les successeurs d'entreprises familiales. Les prédécesseurs ont été éliminés (26 répondants) puisqu'une première analyse des données a permis de constater que plusieurs de leurs réponses différaient systématiquement de celles des successeurs, comportement en accord avec d'autres recherches réalisées auprès de ces deux types d'interlocuteur (Poza, Alfred et Maheshwari, 1997; Sharma, Chrisman et Chua, 2003). De plus, afin de mieux cerner le phénomène de planification du processus dans les entreprises familiales, les données recueillies

4. Traduction libre.

5. Le Centre de recherche industriel du Québec gère une banque de données sur les entreprises québécoises de fabrication, de distribution en gros et de services aux entreprises. L'inscription des entreprises se fait sur une base volontaire et sans frais. En janvier 2002, 26803 entreprises étaient inscrites à ce fichier, soit 13384 entreprises manufacturières, 7624 grossistes et distributeurs et 5795 entreprises de service.

Revue internationale P.M.E., vol. 18, nºs 3-4, 2005

(C) 2005 - Presses de l'Université du Québec

Édifice Le Delta I, 2875, boul. Laurier, bureau 450, Sainte-Foy, Québec G1V 2M2 • Tél.: (418) 657-4399 - www.puq.ca

Tiré de: Revue internationale P.M.E., vol. 18, nos 3-4, sous la direction de Louis Raymond P PME1803N

Tous droits de reproduction, de traduction et d'adaptation réservés 
auprès des entreprises non familiales (32 répondants) n'ont pas été incluses dans cette analyse. Ont été considérées comme familiales les entreprises dont la transmission la plus récente avait été effectuée à l'intérieur de la famille, c'est-à-dire celles que le successeur (le répondant) a acquises d'un membre de sa famille (père, mère, tante, oncle, etc.).

\subsection{Collecte des matériaux et présentation de l'échantillon}

Les données ont été recueillies à l'aide d'un questionnaire ${ }^{6}$ constitué de 50 questions en majorité fermées et divisé en quatre sections: 1) situation actuelle de l'entreprise en ce qui a trait au partage de la direction ou de la propriété; 2) stratégies utilisées pour effectuer la transmission de la propriété et de la direction incluant les activités de planification; 3 ) difficultés éprouvées au cours de la transmission et par la suite et 4) appréciation du succès de ce dernier. Cet article fait principalement référence aux données reliées au processus de transmission de la direction.

Parmi les 115 entreprises dont le prédécesseur ou le successeur a répondu au questionnaire, 65 étaient des entreprises manufacturières (57\%), 44 étaient des grossistes et des distributeurs (39\%) et cinq étaient des entreprises de services $(4 \%)^{7}$. Les répondants occupaient les plus hautes fonctions de l'entreprise (président, PDG, DG) dans $82 \%$ des cas, des postes clés à la trésorerie et aux finances (9\%), à la vice-présidence ou à la direction d'un secteur (3\%) et $6 \%$ occupaient des postes divers; $88 \%$ étaient des hommes. L'âge moyen des répondants était de 46 ans.

Ces entreprises étaient présentes dans 15 des 17 régions administratives du Québec. La représentation régionale est satisfaisante quoique la région de Montréal, qui constitue $24 \%$ de l'échantillon final, soit un peu sous-représentée par rapport à l'échantillon de départ. Quarante-quatre pour cent de ces entreprises embauchaient de 10 à 25 employés, $26 \%$ de 26 à 50 employés et $30 \%, 51$ employés et plus. Quarante-trois pour cent d'entre elles avaient un chiffre d'affaires variant entre un et cinq millions de dollars et $29 \%$, entre 5 et 10 millions de dollars. Les entreprises avaient vécu un transfert de propriété au moins une fois puisque $69 \%$ d'entre elles étaient de deuxième génération et $31 \%$ étaient de troisième génération ou plus.

6. L'enquête téléphonique a été effectuée du début du mois d'avril à la fin du mois de juin 2002. À l'occasion, et à la demande du dirigeant, le questionnaire a été envoyé par télécopieur ou par courrier électronique. Dans tous les cas, l'interviewer devait, dans un premier temps, s'assurer de parler au dirigeant lui-même.

7. La faible représentation des services s'explique non seulement par un taux de réponse plus faible des répondants mais surtout par l'élimination a priori de plusieurs de ces entreprises qui ne répondaient pas aux critères de taille et d'âge.

Revue internationale P.M.E., vol. 18, n ${ }^{\text {os }} 3-4,2005$

(C) 2005 - Presses de l'Université du Québec

Édifice Le Delta I, 2875, boul. Laurier, bureau 450, Sainte-Foy, Québec G1V 2M2 - Tél.: (418) 657-4399 - www.puq.ca

Tiré de: Revue internationale P.M.E., vol. 18, nos $3-4$, sous la direction de Louis Raymond P PME1803N

Tous droits de reproduction, de traduction et d'adaptation réservés 
Le temps écoulé depuis que la transmission avait effectivement eu lieu était variable. Certaines transmissions s'étaient produites à l'intérieur des cinq dernières années (27\%), d'autres dataient de 6 à 10 ans (23\%) et 50\% étaient âgées de plus de 10 ans. Soulignons dans un premier temps que toutes les données présentées dans cet article, sauf une ${ }^{8}$, se rapportent à des faits et non à des impressions, ce qui diminue les modifications de perception qui se produisent souvent avec le passage du temps. De plus, à l'exception de deux variables ${ }^{9}$, aucun biais n'a été décelé dans les données selon que la transmission était âgée de plus de cinq ans ou non.

\section{Résultats}

\subsection{Réussite de la transmission}

Avant d'analyser les activités de planification adoptées par les répondants à la lumière des «meilleures pratiques», il importe d'examiner la performance de leur entreprise depuis la transmission. Toutes ces entreprises ont accompli avec succès au moins une transmission d'entreprise. Dès lors, si la performance est définie en termes de survie/échec, on peut conclure à une performance positive puisque l'échantillon ne comprend aucun cas d'échec confirmé.

Cependant, au-delà du concept de survie, la notion de succès gagne à être développée davantage. Comme en témoignent un certain nombre de recherches menées sur la performance des entreprises familiales, plusieurs indicateurs peuvent être utilisés pour mesurer la réussite, certains financiers mais d'autres personnels et, par conséquent, subjectifs (Sharma, 2004). Dans leur article cherchant à mesurer l'impact de la famille et de l'entreprise sur la viabilité de l'entreprise familiale, Olson et al. (2003) utilisent la croissance du chiffre d'affaires comme mesure objective de la performance de l'entreprise et la perception du succès comme mesure subjective. Dans le cadre d'une recherche portant sur les facteurs de réussite des transmissions d'entreprise, Morris et al. (1997) n'ont, quant à eux, qu'utilisé les critères financiers suivants: croissance du chiffre d'affaires, croissance du bénéfice, croissance du nombre d'employés, croissance des actifs et développement de nouveaux marchés.

Dans la présente recherche, quatre indicateurs financiers (indicateurs objectifs) et un indicateur subjectif ont été utilisés pour cerner la performance de

8. Il s'agit de la question sur la perception du succès de la transmission. La question se formulait comme suit: «Oui ou non, le transfert a-t-il été un succès? Pourquoi?»

9. Il s'agit des deux variables suivantes: le niveau de scolarité des successeurs (qui est plus faible dans le cas des transmissions les plus âgées) et le développement de nouveaux marchés depuis la transmission (qui sont plus fréquents dans le cas des transmissions les plus âgées). Ces effets sont repris dans l'analyse, lorsque pertinents.

Revue internationale P.M.E., vol. 18, n $3-4,2005$

(C) 2005 - Presses de l'Université du Québec

Édifice Le Delta I, 2875, boul. Laurier, bureau 450, Sainte-Foy, Québec G1V 2M2 • Tél.: (418) 657-4399 - www.puq.ca

Tiré de: Revue internationale P.M.E., vol. 18, nos $3-4$, sous la direction de Louis Raymond - PME1803N

Tous droits de reproduction, de traduction et d'adaptation réservés 
l'entreprise après la transmission. Les indicateurs financiers sont: la croissance du chiffre d'affaires et celle du nombre d'employés depuis la transmission, l'évaluation de la santé financière actuelle de l'entreprise et le développement de nouveaux marchés. La mesure subjective fait appel à l'appréciation globale de l'expérience du répondant: «Oui ou non, le transfert a-t-il été un succès?» Cette notion subjective rejoint celle de «satisfaction face au déroulement du processus de transmission» évoquée par Sharma et al. (2001).

Les résultats obtenus pour les cinq indicateurs choisis témoignent du succès des entreprises au regard du processus de transmission. En effet, en ce qui a trait aux critères financiers, les deux tiers ont connu une croissance du nombre d'employés et près des trois quarts ont vu leur chiffre d'affaires augmenter depuis que la transmission a été effectuée. Près de $90 \%$ d'entre elles ont expérimenté une croissance de l'un ou l'autre de ces deux indicateurs. De plus, l'évaluation de la santé financière actuelle de l'entreprise par les répondants est largement positive (92\%), ce qui vient renforcer l'aspect positif de la croissance mesurée par les deux indicateurs précédents. Il est bien connu que la croissance peut avoir des conséquences négatives sur la santé financière d'une entreprise, tout au moins pour les liquidités. Or, seulement $2 \%$ des entreprises qui ont connu une croissance de leur chiffre d'affaires ou du nombre de leurs employés rapportent une situation financière relativement mauvaise. Enfin, un nombre important d'entreprises (70\%) ont réussi à développer de nouveaux marchés. Cependant, comme une différence significative a été constatée pour cette dernière variable en fonction de l'âge de la transmission, le développement de nouveaux marchés étant plus important dans les cas où les transmissions étaient les plus anciennes, l'interprétation de cette variable à titre d'indicateur de la performance est délicate. Quant à la mesure subjective du succès de la transmission, elle est aussi largement positive puisque $87 \%$ des répondants ont une perception positive de la transmission. Parmi les répondants ayant fourni des commentaires sur leur perception du succès, $20 \%$ ont évoqué la situation familiale (p. ex., harmonie dans les rapports familiaux, continuité de l'entreprise dans la famille), $37 \%$, la réussite financière (p. ex., croissance du chiffre d'affaires, de la rentabilité) et $43 \%$, le déroulement du processus lui-même (p. ex., transmission effectuée avec douceur, facilement, dans le respect mutuel). Comme l'ensemble des résultats témoignent d'une performance positive de ces entreprises relativement au processus de transmission à la fois sur les plans objectif et subjectif, l'examen des stratégies des répondants, en ce qui a trait à la planification du processus, est donc susceptible d'apporter un éclairage intéressant sur les mérites des activités de planification.

\subsection{Activités de planification adoptées}

Dans cette section, les activités de planification adoptées par les répondants sont présentées en suivant les différentes catégories apparaissant à la figure 1.

Revue internationale P.M.E., vol. 18, n ${ }^{\text {os }} 3-4,2005$

(C) 2005 - Presses de l'Université du Québec

Édifice Le Delta I, 2875, boul. Laurier, bureau 450, Sainte-Foy, Québec G1V 2M2 - Tél.: (418) 657-4399 - www.puq.ca

Tiré de: Revue internationale P.M.E., vol. 18, nos $3-4$, sous la direction de Louis Raymond - PME1803N

Tous droits de reproduction, de traduction et d'adaptation réservés 
En ce qui a trait à l'établissement de règles de base (première étape du processus dans la figure 1, deux éléments sont examinés, soit l'existence: 1) de critères de choix du successeur, 2) de documents écrits sur la vision d'avenir de l'entreprise. Pour les critères de choix du successeur, on constate que, pour une très grande partie des répondants $(82 \%)$, des critères de choix spécifiques existaient. Ces critères étaient en majorité basés sur les compétences, les qualifications et la motivation des successeurs quoique pour près de $40 \%$ d'entre eux, le critère de choix faisait intervenir une dimension familiale (p. ex., choisir l'aîné de la famille, choisir parmi les enfants intéressés). De plus, dans $83 \%$ des cas où des critères étaient présents, les successeurs en avaient été informés. Ces résultats témoignent d'un souci de planification du processus de transmission: non seulement dans la plupart des cas des critères de sélection existaient mais les principaux intéressés, les successeurs, étaient aussi au courant de ces critères.

Les résultats sont beaucoup moins probants en ce qui a trait à l'existence de documents écrits sur la vision d'avenir de l'entreprise puisque seulement $12 \%$ des répondants ont mentionné avoir utilisé un tel outil de planification. Cette absence de documents écrits ne signifie pas pour autant que les successeurs ne s'étaient pas fixés d'objectifs au moment d'accéder à la direction de l'entreprise: parmi ceux qui n'avaient pas de tels documents, $65 \%$ étaient en mesure de décrire le défi principal qu'ils s'étaient fixé à ce moment-là. La nature de ces défis est variée. L'objectif de croissance du chiffre d'affaires est le plus important (40\% à lui seul), mais les défis de gestion, financiers ou autres, sont aussi nombreux (améliorer la qualité, renouveler les équipements, augmenter la profitabilité, etc.).

Au regard de la préparation du successeur (deuxième étape du processus dans la figure 1 , on constate qu'une majorité de répondants $(70 \%)$ ont complété des études de niveau collégial ou universitaire ${ }^{10}$. Cependant, seule une faible proportion (27\%) de ceux ayant poursuivi des études au-delà du secondaire a fait son choix d'études en vue d'assumer un jour la direction de l'entreprise. De plus, si 53\% des répondants ont bénéficié d'une expérience de travail à l'extérieur de l'entreprise, cette proportion tombe à $44 \%$ en excluant les sujets pour lesquels cette expérience est inférieure à une année. Quant au niveau d'entrée dans l'entreprise, la grande majorité des participants à l'enquête s'y est joint en assumant d'abord des postes de base (manœuvre, journalier, chauffeur, commis, etc.) ou intermédiaires (technicien, contremaître, vendeur, etc.), ce qui suppose un cheminement à l'interne qui donne au successeur l'occasion d'être exposé à divers aspects du fonctionnement de l'entreprise. Finalement, seulement le quart d'entre eux n'ont pas bénéficié de moyens complémentaires de formation. Parmi ces autres moyens de formation,

10. Ce pourcentage est encore plus élevé, soit 79\%, chez les répondants dont la transmission remonte à moins de cinq ans.

Revue internationale P.M.E., vol. 18, nºs 3-4, 2005

(C) 2005 - Presses de l'Université du Québec

Édifice Le Delta I, 2875, boul. Laurier, bureau 450, Sainte-Foy, Québec G1V 2M2 • Tél.: (418) 657-4399 - www.puq.ca

Tiré de: Revue internationale P.M.E., vol. 18, nos $3-4$, sous la direction de Louis Raymond • PME1803N

Tous droits de reproduction, de traduction et d'adaptation réservés 
l'octroi de mandats spéciaux est la technique de formation la plus largement utilisée, suivi du coaching par une personne de l'interne, puis du coaching par une personne de l'extérieur.

L'examen des parcours suivis pour arriver à la direction est aussi révélateur du degré de préparation des successeurs dans les entreprises répondantes. Par exemple, tous ceux qui sont entrés directement à un poste de direction bénéficiaient d'une expérience à l'extérieur de l'entreprise supérieure à une année et la majorité d'entre eux avait complété des études postsecondaires. Quant aux répondants qui sont entrés dans l'entreprise à un poste de base ou de niveau intermédiaire, bien qu'une forte majorité ait complété des études collégiales ou universitaires, plusieurs d'entre eux n'avaient aucune expérience à l'extérieur de l'entreprise, ce qui peut expliquer en partie le fait qu'ils n'ont pas accédé directement à un poste de direction. Toutes ces personnes sans expérience extérieure ont par ailleurs profité de moyens complémentaires de formation.

La planification de la troisième étape du processus est caractérisée dans la figure 1 par la prévision d'un rôle pour le prédécesseur après la transmission de la direction et par la fixation d'une date pour son départ. À cet égard, les résultats sont les suivants. Un rôle satisfaisant pour le prédécesseur avait été prévu pour seulement la moitié des prédécesseurs vivants au moment de la transmission. Une proportion encore plus faible d'entre eux, soit le quart, avait fixé à l'avance une date précise de départ. Il est intéressant d'examiner les rôles assumés par les prédécesseurs: alors que $28 \%$ d'entre eux se sont retirés complètement, $25 \%$ assument le rôle de président du conseil d'administration, le reste occupant des rôles variés (responsable des ventes, des relations publiques, de la qualité, vendeur, conseiller, etc.).

Finalement, en ce qui a trait aux deux éléments contextuels de la figure 1 (contextes de l'entreprise et de la famille), on constate la présence d'un conseil d'administration dans près de la moitié des cas (42\%), quoique cette proportion tombe à moins de $20 \%$, si l'on tient compte de la présence de membres extérieurs à la famille ou à l'entreprise au conseil. Qui plus est, seulement la moitié de ces répondants reconnaissent un rôle actif ${ }^{11}$ au conseil d'administration dans le choix du successeur. Le conseil de famille est aussi peu présent (24\%). Il en est de même de la prévision de la place des conjoints dans l'entreprise (28\%).

11. Ces répondants ont décrit le rôle du conseil d'administration dans les termes suivants: grande implication, rôle ultime, rôle décisif, rôle prépondérant, rôle de confirmation, sounding board.

Revue internationale P.M.E., vol. 18, n ${ }^{\text {os }} 3-4,2005$

(C) 2005 - Presses de l'Université du Québec

Édifice Le Delta I, 2875, boul. Laurier, bureau 450, Sainte-Foy, Québec G1V 2M2 - Tél.: (418) 657-4399 - www.puq.ca

Tiré de: Revue internationale P.M.E., vol. 18, nos $3-4$, sous la direction de Louis Raymond P PME1803N

Tous droits de reproduction, de traduction et d'adaptation réservés 


\subsection{Discussion}

Les activités de planification recensées chez les répondants sont maintenant comparées aux «meilleures pratiques»; le tableau 1 fait état de cette comparaison. En ce qui concerne les activités reliées à l'établissement des règles de base, plus particulièrement pour l'existence de critères de choix du successeur et la communication à l'avance de ces critères, on peut avancer que l'échantillon présente un haut degré de conformité avec les «meilleures pratiques». Ce n'est pas le cas pour la présence de documents écrits sur la vision d'avenir de l'entreprise. Les défis que s'étaient fixés les successeurs au moment du transfert ont pu compenser pour cette lacune. Autrement dit, ce n'est pas parce qu'il n'y avait pas de documents écrits pour soutenir la vision que cette dernière n'existait pas.

Tableau 1

Sommaire des activités recensées

\begin{tabular}{|c|c|c|}
\hline Activités recensées & 异 & $\begin{array}{c}\text { Explication possible de l'écart } \\
\text { par rapport aux } \\
\text { «meilleures pratiques» }\end{array}$ \\
\hline \multicolumn{3}{|c|}{ Activités reliées à l'établissement des règles de base } \\
\hline Existence de critères de choix du successeur. & $82 \%$ & \\
\hline $\begin{array}{l}\text { Connaissance à l'avance } \\
\text { des critères de choix. }\end{array}$ & $68 \%$ & \\
\hline $\begin{array}{l}\text { Existence de documents écrits sur } \\
\text { la vision d'avenir de l'entreprise. }\end{array}$ & $12 \%$ & $\begin{array}{l}\text { Compensation par la présence } \\
\text { de défis identifiés. }\end{array}$ \\
\hline \multicolumn{3}{|c|}{ Activités reliées à la préparation du successeur } \\
\hline Études de niveau postsecondaire complétées. & $70 \%$ & \multirow{4}{*}{$\begin{array}{l}\text { Compensation par le niveau d'entrée, de } \\
\text { scolarité et d'autres moyens de formation. }\end{array}$} \\
\hline $\begin{array}{l}\text { Entrée à un poste de base ou de niveau } \\
\text { intermédiaire. }\end{array}$ & $78 \%$ & \\
\hline $\begin{array}{l}\text { Présence d'expérience de travail à l'extérieur } \\
\text { de l'entreprise. }\end{array}$ & $53 \%$ & \\
\hline Utilisation d'autres moyens de formation. & $75 \%$ & \\
\hline \multicolumn{3}{|c|}{ Activités reliées au désengagement du prédécesseur } \\
\hline Rôle (satisfaisant) prévu pour le prédécesseur. & $50 \%$ & Effet de taille et de génération*. \\
\hline Date fixée pour le départ du prédécesseur. & $24 \%$ & Faible conformité - aucune explication. \\
\hline \multicolumn{3}{|c|}{ Activités reliées aux contextes de la famille et de l'entreprise } \\
\hline Présence d'un CA avant la transmission. & $42 \%$ & Effet de taille** et de génération*. \\
\hline Présence d'un conseil de famille. & $24 \%$ & Effet de taille**. \\
\hline $\begin{array}{l}\text { Prévision de la place des conjoints } \\
\text { dans l'entreprise. }\end{array}$ & $28 \%$ & Faible conformité - aucune explication. \\
\hline
\end{tabular}

* $\mathrm{p}<10 \%$, test de Kruskal-Wallis.

** $\mathrm{p}<5 \%$, test de Kruskal-Wallis.

Revue internationale P.M.E., vol. 18, $\mathrm{n}^{\text {os }} 3-4,2005$

(C) 2005 - Presses de l'Université du Québec

Édifice Le Delta I, 2875, boul. Laurier, bureau 450, Sainte-Foy, Québec G1V 2M2 - Tél.: (418) 657-4399 - www.puq.ca

Tiré de: Revue internationale P.M.E., vol. 18, nos 3-4, sous la direction de Louis Raymond • PME1803N

Tous droits de reproduction, de traduction et d'adaptation réservés 
Relativement à la préparation des successeurs, on retrouve également une forte présence de plusieurs de ces activités chez les répondants et on peut en conclure que la majorité d'entre eux se conforment aux meilleures pratiques. Même s'il peut sembler paradoxal que certains répondants ne s'y soient pas conformés et aient tout de même réussi à transmettre avec succès leur entreprise, l'examen des cheminements de préparation des successeurs est révélateur. À titre d'exemple, ceux qui sont entrés à un poste de direction ont compensé pour ce comportement qui s'éloigne des «meilleures pratiques» par une expérience de travail à l'extérieur de l'entreprise d'une durée suffisante à laquelle se sont ajoutées, dans la plupart des cas, des études postsecondaires et l'utilisation de certains moyens complémentaires de formation. D'autre part, les successeurs qui ne détenaient qu'un diplôme d'études secondaires (DES) sont entrés, pour la très grande majorité, à un poste de base ou de niveau intermédiaire et ont fait appel à des moyens complémentaires de formation. En considérant la totalité de l'échantillon, une seule personne n'a observé aucune des «meilleures pratiques» en ce qui a trait à la formation du successeur: elle ne possédait qu'un DES, n'avait pas d'expérience à l'extérieur de l'entreprise, n'avait fait appel à aucun moyen complémentaire de formation et était entrée à un poste de direction. Elle constitue, en quelque sorte, l'exception qui confirme la règle.

Pour terminer l'analyse sur la préparation des successeurs, il faut souligner que la présence d'expérience de travail à l'extérieur de l'entreprise est la seule variable de cette catégorie qui est présente chez à peine plus de $50 \%$ des répondants. Par ailleurs, ni la taille des entreprises, ni le nombre de générations, ni l'âge de la transmission n'ont pu être associés à ce comportement.

S'agissant du désengagement du prédécesseur, le comportement des répondants s'éloigne des «meilleures pratiques», et ce, relativement aux deux variables mesurées dans cette catégorie d'activités. Ces résultats rejoignent ceux de Sharma, Chua et Chrisman (2003) dans leur étude sur la planification de la succession et questionnent le statut de «meilleures pratiques» de ces activités. Une explication peut venir de la prise en compte de la taille des entreprises. En effet, on constate un effet de taille dans le cas de la prévision d'un rôle satisfaisant pour le prédécesseur après l'entrée en poste du successeur, c'est-à-dire que plus les entreprises sont grandes, plus elles se conforment à la «meilleure pratique» à cet égard. Cela tient peut-être au fait que dans les entreprises plus petites, il est plus facile de trouver un rôle satisfaisant pour le prédécesseur dans un délai très court: l'organisation peut faire preuve de plus de souplesse et les besoins sont souvent très grands. On constate aussi un effet de génération, c'est-à-dire que les entreprises de troisième génération ou plus se conforment plus que les autres; dans ces cas, ce peut être l'expérience qui entre en ligne de compte. Les prédécesseurs des entreprises de troisième génération, ayant déjà vécu une transmission dans le siège du successeur, sont plus enclins à

Revue internationale P.M.E., vol. 18, $\mathrm{n}^{\text {os }} 3-4,2005$

(C) 2005 - Presses de l'Université du Québec

Édifice Le Delta I, 2875, boul. Laurier, bureau 450, Sainte-Foy, Québec G1V 2M2 - Tél.: (418) 657-4399 - www.puq.ca

Tiré de: Revue internationale P.M.E., vol. 18, nos $3-4$, sous la direction de Louis Raymond P PME1803N

Tous droits de reproduction, de traduction et d'adaptation réservés 
penser à préparer leur sortie. Ces deux effets ne se retrouvent pas en ce qui a trait à l'existence d'une date fixée à l'avance pour le départ du prédécesseur et cet écart par rapport aux «meilleures pratiques» demeure sans explication.

Relativement aux structures de gouvernance, force est de constater que l'absence de conformité aux «meilleures pratiques» ne semble pas avoir nui aux participants à l'étude. Dans le cas du conseil d'administration, l'effet de taille joue fortement, les plus grandes entreprises étant plus nombreuses à fonctionner avec un tel conseil. L'effet de génération joue également: les entreprises de troisième génération ou plus sont aussi plus nombreuses à bénéficier des services d'un conseil d'administration. On peut avancer l'hypothèse que, dans les plus petites, l'absence d'un tel conseil ne constitue pas un handicap important pour mener à terme un processus de transmission, les plus petites entreprises n'éprouvant pas encore le besoin de formaliser leur gestion. Par ailleurs, l'effet de taille joue au regard de la présence du conseil de famille. Encore une fois, pour les entreprises plus petites, un conseil de famille formel ne semble pas essentiel à la réussite du processus. Ces derniers constats n'excluent cependant pas le fait que le processus pourrait être plus harmonieux si ces structures étaient présentes. Finalement, en ce qui a trait à la pratique consistant à prévoir la place des conjoints dans l'entreprise, aucun effet de taille ou de génération ne semble expliquer sa faible fréquence dans l'échantillon.

\section{Conclusion}

L'étude réalisée a permis d'explorer le comportement de 115 PME familiales québécoises au regard de leur planification du processus de transmission de la direction au sein de leur entreprise. Les activités de planification relatives à la planification stratégique, à la préparation des successeurs et au désengagement du prédécesseur ont été examinées. Des éléments contextuels, comme la présence de certaines structures de gouvernance, ont également été inclus dans l'analyse.

L'examen des résultats nous apprend que les activités relatives à la préparation du successeur, celles concernant l'établissement de critères de choix du successeur et la communication de ces critères aux principaux intéressés, ont été suffisamment nombreuses pour avancer que les PME familiales québécoises se conforment aux «meilleures pratiques» en ce domaine. Le même constat ne peut cependant pas être fait pour les activités relatives au désengagement du prédécesseur, pour celles reliées à la planification stratégique dans un sens strict (c'est-à-dire appuyée par l'existence de documents écrits sur la vision d'avenir de l'entreprise), ni pour l'existence des structures de gouvernance telles que le conseil d'administration et le conseil de famille. Dans ces cas, le comportement des entreprises répondantes apparaît très éloigné de celui dicté par les «meilleures pratiques».

Revue internationale P.M.E., vol. 18, nºs 3-4, 2005

(C) 2005 - Presses de l'Université du Québec

Édifice Le Delta I, 2875, boul. Laurier, bureau 450, Sainte-Foy, Québec G1V 2M2 • Tél.: (418) 657-4399 - www.puq.ca

Tiré de: Revue internationale P.M.E., vol. 18, nos 3-4, sous la direction de Louis Raymond P PME1803N

Tous droits de reproduction, de traduction et d'adaptation réservés 
Comme toutes les entreprises de l'échantillon ont réussi au moins un transfert intergénérationnel, faut-il en conclure que ces activités, qualifiées dans la littérature de «meilleures pratiques», ne sont pas essentielles à la réussite?

La réponse à cette question demande de mettre en lumière les limites de l'étude réalisée. En premier lieu, la mesure de la réussite gagnerait à être plus nuancée. Les indicateurs de succès recueillis dans cette étude n'ont pas permis d'apporter cette nuance et très peu de liens statistiquement significatifs ont pu être établis entre les activités de planification réalisées par les répondants et ces indicateurs. Plusieurs facteurs peuvent expliquer cette situation. En premier lieu, les variables qui influent sur la performance financière sont nombreuses et il est difficile d'en isoler les effets spécifiques des activités de planification. De plus, qui dit réussite ne dit pas nécessairement parcours sans difficultés. Le degré de difficultés éprouvées à la suite de la transmission des pouvoirs à la génération suivante pourrait servir à nuancer la notion de succès. Si ce niveau de difficultés peut être expliqué en partie par la présence ou non de ces activités de planification, leur classement parmi les «meilleures pratiques» prendrait alors tout son sens.

Le fait de ne pas avoir considéré l'ensemble des activités de planification reliées à la transmission de la direction constitue également une limite de cette étude. À cet égard, l'examen des activités reliées à la préparation du successeur a bien fait ressortir l'avantage de considérer, non pas les activités réalisées une à une, mais de façon intégrée. Cette d'analyse a permis d'observer un phénomène de compensation entre les divers moyens utilisés pour sa formation. De la même manière, certaines activités non répertoriées dans cette recherche ont pu être réalisées par les entreprises répondantes et ont pu compenser, par exemple, pour l'absence d'un conseil d'administration et de famille.

Malgré ces limites, cette recherche apporte de nouvelles connaissances sur le phénomène de la planification du processus de transmission d'entreprises en contexte de PME. Elle fait ressortir, entre autres, la souplesse dont peuvent faire preuve les entreprises dans la gestion de la relève à la haute direction. Elle souligne aussi la nécessité de tenir compte, comme l'ont mentionné Le Breton-Miller et Miller (2004), du contexte de l'entreprise, entre autres de sa taille, afin de relativiser la liste des «meilleures pratiques».

Plusieurs avenues de recherche se dessinent dans la suite des résultats de cette étude. La première se rapporte à l'établissement d'outils de mesure de la réussite suffisamment sensibles pour permettre de vérifier l'incidence des activités de planification sur le succès et sur les difficultés avec lesquelles se vit la transmission dans les PME. Cet objectif constitue un véritable défi puisqu'il demande d'avoir accès, entre autres, à des données financières suffisantes auprès d'un échantillon important d'entreprises, ce qui est souvent un problème dans les petites et moyennes entreprises à capital fermé.

Revue internationale P.M.E., vol. 18, $\mathrm{n}^{\text {os }} 3-4,2005$

(C) 2005 - Presses de l'Université du Québec

Édifice Le Delta I, 2875, boul. Laurier, bureau 450, Sainte-Foy, Québec G1V 2M2 - Tél.: (418) 657-4399 - www.puq.ca

Tiré de: Revue internationale P.M.E., vol. 18, nos 3-4, sous la direction de Louis Raymond • PME1803N

Tous droits de reproduction, de traduction et d'adaptation réservés 
À l'instar de Miller, Steier et Le Breton-Miller (2003), l'examen de PME qui ont échoué le processus de transmission permettrait de relativiser les résultats obtenus. Enfin, il est nécessaire de parfaire un modèle intégrateur des activités de planification susceptible d'être testé auprès d'un échantillon important de PME. Ce modèle, s'appuyant sur le travail de Le Breton-Miller et Miller (2004), permettrait de voir la planification du processus dans sa totalité et de mieux comprendre, spécialement en contexte de PME, les activités qui sont essentielles et celles qui peuvent faire l'objet d'une certaine flexibilité.

\section{Bibliographie}

Aronoff, C.E. (2001), «Understanding family-business survival statistics», Supply House Times, juillet, p. 34-35.

BANQUE DE DÉVELOPPEMENT DES PME - BDPME (1998), La transmission des PME-PMI10 années d'expérience de la Banque de développement des PME, Paris, 46 p. Site Web: <www.transmission-pme.ch/etudes.html>.

BARACH, J.A. et J.B. GANTISKY (1995), «Successful succession in family business», Family Business Review, vol. 8, n 2, p. 131-155.

BARACH, J.A., J. GANTisky, J.A. CARson et A. Doochin (1988), «Entry of the next generation: strategic challenge for family business», Journal of Small Business Management, vol. 26, p. 49-56.

Cabrera-Suárez, K., P. De SaA-Pérez et D. García-Almeida (2001), «The succession process from a resource- and knowledge-based view of the family firm», Family Business Review, vol. 14, nº 1, p. 37-46.

CAdieux, L. et J. Lorrain (2004), «Et si assurer la relève dépendait aussi de la manière dont les prédécesseurs réussissent à se désengager?», Gestion, Revue internationale de gestion, vol. 29, $\mathrm{n}^{\circ} 3$, automne, p. 120-128.

Campbell Expertise Marketing (2000), Sondage réalisé sous la direction de Fernand Campbell, «Étude des besoins et de l'intérêt des familles en affaires pour une formation sur la gestion des entreprises familiales». Adresse électronique: <c.e.marketing@ videotron.ca>.

Churchill, N.C. et K.J. Hatten (1987), «Non-market-based transfers of wealth and power: a research framework for family business», American Journal of Small Business, vol. 11, no 3, p. 51-64.

Cliffe, S. (1998), «Family business: facing up to succession», Harvard Business Review, vol. 76, n 3, p. 16-17.

Davis, P.S. et P.D. Harveston (1998), «The influence of family business succession process: a multi-generational perspective», Entrepreneurship: Theory and Practice, p. 31-53.

FILE, P. et R.A. PrinCE (1996), «Attributions for family business failure: the heir perspective», Family Business Review, vol. 9, n 2, p. 171-184.

Revue internationale P.M.E., vol. 18, nºs 3-4, 2005

(C) 2005 - Presses de l'Université du Québec

Édifice Le Delta I, 2875, boul. Laurier, bureau 450, Sainte-Foy, Québec G1V 2M2 • Tél.: (418) 657-4399 - www.puq.ca

Tiré de: Revue internationale P.M.E., vol. 18, nos $3-4$, sous la direction de Louis Raymond - PME1803N

Tous droits de reproduction, de traduction et d'adaptation réservés 
Goldberg, S.D. (1996), «Effective successors in family-owned business», Family Business Review, vol. 9, n² 2, p. 185-197.

HANDLER, W.C. (1990), «Succession in family firms: a mutual role adjustment between entrepreneur and next-generation family members», Entrepreneurship: Theory and Practice, vol. 15, n ${ }^{\circ}$ 1, p. 37-51.

Hugron, P. (1991), L'entreprise familiale. Modèle de réussite du processus successoral, Montréal, Institut des recherches politiques et Presses HEC, XVII-155.

LANSBERg, I.S. (1988), «The succession conspiracy», Family Business Review, vol. 1, nº 2 , p. 119-143.

Le Breton-Miller, I. et D. Miller (2004), «Toward an integrative model of effective FOB succession», Entrepreneurship Theory and Practice, été, p. 305-328.

Longenecker, J.G. et J.E. Schoen (1978), «Management succession in the family business », Journal of Small Business Management, vol. 16, p. 1-6.

Malone, S.C. (1989), «Selected correlates of business continuity planning in the family business», Family Business Review, vol. 2, n 4, p. 341-353.

McGivern, C. (1978), «The dynamics of management succession», Management Decision, vol. $16, \mathrm{n}^{\circ} 1$, p. $32-42$.

Miller, D., L. Steier et I. Le Breton-Miller (2003), «Lost in time: intergenerational succession, change and failure in family business», Journal of Business Venturing, vol. $18, \mathrm{n}^{\circ} 4$, p. 513-551.

Morris, M.H., R.O. Williams, J.A. Allen et R.A. Avila (1997), «Correlates of success in family business transitions», Journal of Business Venturing, vol. 12, p. 385-401.

Murray, B. (2003), «The succession transition process: a longitudinal perspective», Family Business Review, vol. 16, $\mathrm{n}^{\circ}$ 1, p. 17-33.

Olson, P.D., V.S. Zuiker, S.M. Danes, K. Stafford, R.K.Z. Heck et K.A. Duncan (2003), «Impact of family and business on family business sustainability», Journal of Business Venturing, vol. 18, n 5, p. 639-666.

Poza, E.J., T. Alfred et A. MAheshwari (1997), «Stakeholder perceptions of culture and management practices in family firms - A preliminary report», Family Business Review, vol. 10, $\mathrm{n}^{\circ}$ 2, p. 135-155.

Sharma, P. (2004), «An overview of the field of family business studies: current status and directions for the future», Family Business Review, vol. XVII, n ${ }^{\circ}$, mars, p. 1-36.

Sharma, P., J.J. Chrisman et J.H. Chua (2003), «Predictors of satisfaction with the succession process in family firms», Journal of Business Venturing, vol. 18, $\mathrm{n}^{\circ} 5$, p. 667-687.

Sharma, P., J.J. Chrisman, A.L. Pablo et J.H. Chua (2001), «Determinants of initial satisfaction with the succession process in family firms: a conceptual model», Entrepreneurship Theory and Practice, vol. 25, nº 3, p. 17-36.

Sharma, P., J.H. ChuA et J.J. Chrisman (2003), «Succession planning as planned behaviour: some empirical results», Family Business Review, vol. XVI, n 1, p. 1-15.

Revue internationale P.M.E., vol. 18, $\mathrm{n}^{\text {os }} 3-4,2005$

(C) 2005 - Presses de l'Université du Québec

Édifice Le Delta I, 2875, boul. Laurier, bureau 450, Sainte-Foy, Québec G1V 2M2 - Tél.: (418) 657-4399 - www.puq.ca

Tiré de: Revue internationale P.M.E., vol. 18, nos $3-4$, sous la direction de Louis Raymond • PME1803N

Tous droits de reproduction, de traduction et d'adaptation réservés 
St-Cyr, L. et F. Richer (2002), «Défis et stratégies de transmission de PME québécoises Étude exploratoire», Actes du colloque "Les enjeux de la transmission d'entreprise», Groupe d'études en sciences de l'entreprise et des marchés (GESEM) et Faculté d'administration et de gestion, Université de Montpellier I, p. 235-249.

Ward, J.L. (1987), Keeping the Family Business Healthy, San Francisco, Jossey-Bass, $266 \mathrm{p}$.

Revue internationale P.M.E., vol. 18, $\mathrm{n}^{\text {os }} 3-4,2005$

(C) 2005 - Presses de l'Université du Québec

Édifice Le Delta I, 2875, boul. Laurier, bureau 450, Sainte-Foy, Québec G1V 2M2 • Tél.: (418) 657-4399 - www.puq.ca

Tiré de: Revue internationale P.M.E., vol. 18, nos $3-4$, sous la direction de Louis Raymond - PME1803N

Tous droits de reproduction, de traduction et d'adaptation réservés 